\title{
LA PLANIFICACIÓN DE COSECHA EN CIRUELA VARIEDAD HORVIN, ESTUDIO DE CASO. TUTA, BOYACÁ, COLOMBIA*
}

\author{
HARVEST PLANNING IN HORVIN PLUM VARIETY, A CASE \\ STUDY IN TUTA, BOYACÁ, COLOMBIA \\ PLANEAMENTO DA COLHEITA DA AMEIXA NA \\ VARIEDADE HORVIN, ESTUDO DE CASO EM TUTA, \\ BOYACÁ, COLÔMBIA \\ PLANIFICATION DE LA RECOLTE DE PRUNE DE LA \\ VARIETE HORVIN, ETUDE DE CAS DANS TUTA, BOYACA, \\ COLOMBIE
}

\author{
ANA MILENA SERRANO AMADO ${ }^{1}$ \\ GLORIA ACEDEN PUENTES MONTAÑÉZZ ${ }^{2}$ \\ ALEXANDER CORONADO VIASÚS ${ }^{3}$
}

Fecha de recepción: 12 de agosto de 2019

Fecha de aprobación: 15 de octubre de 2020

* Resultado del proyecto de investigación Planificación de Ciruela Horvin en el municipio de Tuta"t Ingeniera industrial, Universidad del Valle. Cali, Valle del Cauca, Colombia Master Kaizen, Distribuidora Nissan. E-mail: alexandra.saenz@correunivalle.edu.co. ORCID 0000-0001-9992-2256

1 Administrador de Empresas, Magister en Administración, Doctorante en Administración, Grupo de Investigación Ceres de la Universidad Pedagógica y Tecnológica de Colombia. Ana.serrano@uptc.edu.co - ORCID 0000-0003-0579-5722

2 Administrador Agrícola, Magister en Ciencias Agrarias, Doctorante en Proyectos, Grupo de Investigación Ceres de la Universidad Pedagógica y Tecnológica de Colombia. Gloria.puentes@uptc.edu.co - ORCID 000-0002-5953-9591

3 Administrador de Empresas Agropecuario, Grupo de Investigación Ceres de la Universidad Pedagógica y Tecnológica de Colombia. Alex.coronado@uptc.edu.co ORCID 0000-0002-7246-1098 
En Colombia el cultivo de cirvela generalmente se establece con fines de comercialización, en zonas comprendidas entre 1.800 y 2.450 m de altitud, donde los principales productores son Boyacá y Cundinamarca (Agronet, 2014). Las diversas variedades de cirvela se caracterizan por su capacidad de adaptación en diferentes altitudes.

La cirvela es una especie que puede vivir por décadas en condiciones óptimas, produciéndose en forma continua en zonas tropicales; se considera un cultivo promisorio, debido a su adaptación. Los municipios que cuentan con mayor producción de ciruela en el departamento de Boyacá son Nuevo Colón, con un rendimiento de 19 t./Ha, que representa $40 \%$ del total producido; seguido por Tibaná y Jenesano, que producen 17 t./Ha, y entre los dos suman $30 \%$ del total producido; sigue Sotaquirá, con un rendimiento de 13 ton/ha. y representa $13 \%$ del total producido; luego aparece Tuta, con 10 t/Ha y registra $10 \%$ de la producción total, y los otros municipios registran el $7 \%$ restante con un rendimiento promedio de 8 t/ha. Según Asohofrucol (2012), en estas producciones se presentan pérdidas de fruta que llegan hasta $30 \%$. Unido a esto se relacionan causas en las áreas de producción, cosecha, trasporte y mercados, como desconocimiento de índices de cosecha, empaques inadecuados, falta de control de temperatura y bajos precios del mercado.

Dado lo anterior, el grupo de investigación CERES desarrolla la investigación en el municipio de Tuta, con el ánimo de realizar una planificación adecuada para el cultivo de cirvela con el propósito de mejorar el proceso productivo y controlar las pérdidas de cosechas. Como metodología se implementa un enfoque cualitativo utilizando una metodología PRA (Participatory Rural Apraissal), con la participación de los beneficiarios en el proceso de diagnóstico y el levantamiento de necesidades.

\section{PALABRAS CLAVE:}

matriz dofa, mercadeo, planificación.

CLASIFICACIÓN JEL:

$\mathrm{M} 31, \mathrm{O} 2$.

\section{ABSTRACT}

In Colombia, plum cultivation is usually established for marketing purposes, in areas between 1800 and $2450 \mathrm{~m}$ altitude, where the main producers are Boyacá and Cundinamarca (Agronet, 2014). The different varieties of plum are characterized by their ability to adapt at different altitudes.

The plum is a species that can live for decades under optimal conditions, occurring continuously in tropical areas, considering it a promising crop, due to its adaptation. The municipalities with the highest production of plum in the Department of Boyacá are Nuevo Colon with a yield of 19 ton/Ha that represents $40 \%$ of the total produced; followed by Tibaná 
and Jenesano that produce 17 ton/Ha and the two they account for $30 \%$ of the total produced; Sotaquirá continues with a yield of 13 ton / ha. and represents the total 13\% produced; then Tuta with 10 ton / Ha and it registers $10 \%$ of the total production and the other municipalities register the remaining $7 \%$ with an average performance of 8 ton / ha. Asohofrucol (2012) in these productions presents fruit losses that reach up to $30 \%$. Linked to this are causes in the area of production, harvest, transportation and markets, such as ignorance of harvest rates, inadequate packaging, lack of temperature control and low market prices.

Given the above, the research group CERES develops research in the municipality of Tuta, with the aim of carrying out an adequate planning for the plum culture, with the purpose of improving the production process and controlling crops loses. Qualitative approach using a PRA (Apraissal Participatory Rural) has been the methodology, with the participation of the beneficiaries in the diagnostic process and the surveying of needs.

Keywords: Dofa matrix, marketing, planning.

Jel Classification: M31, O2.

RESUMO

Na Colômbia, o cultivo de ameixas é geralmente estabelecido para fins de comercialização, em áreas entre 1.800 e $2.450 \mathrm{~m}$ de altitude, onde os principais produtores são Boyacá e Cundinamarca (Agronet, 2014). As diferentes variedades de ameixa são caracterizadas pela sua capacidade de adaptação a diferentes altitudes.

A ameixa é uma espécie que pode viver durante décadas em condições óptimas, produzindo continuamente em zonas tropicais; é considerada uma cultura promissora, devido à sua adaptação. Os municípios que têm maior produção de ameixa no departamento de Boyacá são Nuevo Colón, com um rendimento de 19 t./ $\mathrm{Ha}$, que representa $40 \%$ do total produzido; seguidos de Tibaná e Jenesano, que produzem 17 t. / $/ \mathrm{Ha}, \mathrm{e}$ entre os dois representam $30 \%$ da produção total, seguido de Sotaquirá, com um rendimento de 13 t/ha, e representa $13 \%$ da produção total, depois vem Tuta, com 10 t/ha e regista $10 \%$ da produção total, e os outros municípios registaram os restantes $7 \%$ com um rendimento médio de 8 t/ha. Segundo a Asohofrucol (2012), nestas produções há perdas de frutos que chegam a atingir $30 \%$. Isto está relacionado com causas nas áreas de produção, colheita, transporte e mercados, tais como falta de conhecimento dos índices de colheita, embalagem inadequada, falta de controlo da temperatura e baixos preços de mercado.

Tendo em conta o acima exposto, o grupo de investigação CERES desenvolve a investigação no município de Tuta, com o objectivo de fazer um planeamento adequado para o cultivo de ameixas a fim de melhorar o processo de produção e controlar as perdas de colheitas. Como metodologia, é implementada uma abordagem qualitativa utilizando uma metodologia PRA (Participatory Rural Appraisal), com a participação dos beneficiários no processo de diagnóstico e avaliação das necessidades.

Palavras-chave: marketing; matriz dofa; planeamento.

Classificação JEL: M31, O2. 
En Colombie, la culture de la prune est généralement établie à des fins de commercialisation, dans des zones situées entre 1.800 et $2.450 \mathrm{~m}$ d'altitude, où les principaux producteurs sont Boyacá et Cundinamarca (Agronet, 2014). Les différentes variétés de prunes se caractérisent par leur capacité d'adaptation à différentes altitudes.

La prune est une espèce qui peut vivre pendant des décennies dans des conditions optimales, produisant continuellement dans les zones tropicales; il est considéré comme une culture prometteuse, en raison de son adaptation. Les municipalités qui ont une plus grande production de prunes dans le département de Boyacá sont Nuevo Colón, avec un rendement de 19 t./ $/ \mathrm{Ha}$, ce qui représente $40 \%$ du total produit; suivies de Tibaná et Jenesano, qui produisent 17 t. / Ha et entre les deux représentent $30 \%$ de la production totale, suivie par Sotaquirá, avec un rendement de 13 tonnes / ha, et représente 13\% de la production totale, puis vient Tuta, avec 10 † / ha et enregistre $10 \%$ de la production totale, et les autres municipalités ont enregistré les $7 \%$ restants avec un rendement moyen de 8 t / ha. Selon Asohofrucol (2012), dans ces productions il y a des pertes de fruits qui atteignent jusqu'à $30 \%$. Cette situation est liée à des causes dans les domaines de la production, de la récolte, du transport et des marchés, telles que la méconnaissance des indices de récolte, l'emballage inadéquat, le manque de contrôle de la température et les faibles prix du marché.

Compte tenu de ce qui précède, le groupe de recherche CERES développe la recherche dans la municipalité de Tuta, dans le but de faire une planification adéquate pour la culture des prunes afin d'améliorer le processus de production et de contrôler les pertes de récolte. Comme méthodologie, une approche qualitative est mise en œuvre en utilisant une méthodologie PRA (Participatory Rural Appraisal), avec la participation des bénéficiaires dans le processus de diagnostic et d'évaluation des besoins.

Mots clés: matrice dofa, marketing, planification.

Classification JEL: M31, O2.

\section{INTRODUCCIÓN}

La economía colombiana creció 2,8\%. En el caso del sector de agricultura, ganadería, caza, silvicultura y pesca el PIB se incrementó $5,9 \%$. No obstante, el sector agropecuario, silvícola y pesquero tiene un peso medio en la economía nacional, pues ocupó el séptimo lugar entre 12 sectores, en la participación del PIB total de la economía, con $6,9 \%$, en el PIB del segundo trimestre de 2018. El café sigue siendo uno de los principales jalonadores de la economía agrícola y durante el segundo trimestre de 2018 la producción cafetera nacional presentó un comportamiento positivo, al alcanzar 2,9 millones de sacos, lo que significa un crecimiento de 5,2\% (Departamento Administrativo Nacional de Estadística, DANE, 2018). En el sector de Boyacá según el informe de 2016, el sector agropecuario ha crecido y se repunta en el Departamento, ya que el aporte del sector al PIB pasó de registrar $15.3 \%$ en 2015 a $17.3 \%$ en 2017 , demostrando así que las iniciativas del 
gobierno departamental han dado resultado (Gobernación de Boyacá, 2017), y aunque en el departamento de Boyacá la mayoría de las producciones son en cultivos transitorios y perenes, los cultivos ornamentales no aportan en el PIB.

Según estadísticas de (Agronet, 2019), en Colombia para el año 2018 la producción de cirvela fue de 18.028.26 ton, ocupando 1.361.3 ha, con un rendimiento de 13.24 (ton / ha), por lo cual se establece una variación significativa en cuanto al año anterior debido a que la producción promedio fue de 18.091.66 ton con un rendimiento de 13.53 (ton / ha).

En el departamento de Boyacá se destaca que para el año 2018 se cosecharon 1.048, 60 (ha) con un promedio de 12.753 .6 ton en cuanto a producción, lo cual se considera importante para el sector debido a su relevancia; por ello para Puentes (2006), Boyacá se considera el departamento más importante en la explotación de los cultivos de caducifolios en el país, debido a las ventajas comparativas que posee, entre las cuales se pueden mencionar: clima, suelos, precipitación, acumulación de horas frío, vocación frutícola de los productores y experiencia acumulada por más de 50 años.

La cirvela es una especie que puede vivir por décadas en condiciones óptimas, produciéndose de manera continua en zonas tropicales. La cirvela se considera un cultivo promisorio para Boyacá y otros departamentos en Colombia, debido a su adaptación en zonas comprendidas entre 2.100 y $2.600 \mathrm{~m}$ de altitud (Orjuela, 2016). Así mismo, entre los municipios productores de cirvela para el año 2018 se encuentra Nuevo Colón, con una producción de 5.400 ton y un rendimiento de 12,00 (ton / ha); Sotaquirá, con un promedio de 2.210 ton y rendimiento de 14,00 (ton / ha); Jenesano con 1.680 ton y 12,00 (ton / ha); Tibaná con una producción de 1.484 y un rendimiento de 14,00 (ton / ha), y finalmente Tuta, con 255 (ton) y rendimiento promedio de 12,00 (ton / ha).

Las unidades productivas de caducifolios en el departamento de Boyacá reúnen las siguientes características: área en promedio de 3,72 ha, altas pendientes $18,29 \%$ en $62 \%$ de las fincas, con mínimo 0,48 ha de la finca dedicada a este cultivo, 133 árboles/finca, edad promedio de 7,79 años, rendimiento de 27,47 ton /ha/año.

Los agricultores son en su mayoría

arrendatarios, complementan esta actividad con otras especies frutícolas y otras acciones, con disponibilidad de riego localizado, hacen análisis de suelos y no utilizan compensadores de frío.

En la actualidad la importancia de Boyacá en la explotación de cultivos de caducifolios en el sector nacional ha tenido avances importantes en lo que respecta a manejo empresarial, introducción de nuevas variedades, innovaciones tecnológicas, infraestructura de riego, mentalidad de trabajo asociativo, mayores extensiones, planificación de cultivos teniendo en cuenta los requerimientos del mercado, y el trabajo bajo el enfoque de cadena productiva. Una práctica que se constituye en factor fundamental para que la explotación de caducifolios sea rentable es la programación de las cosechas, práctica que consiste en obligar a los frutales a producir en épocas diferentes a las comunes mediante el manejo de variables de producción (Puentes, Prieto y Caro, 2019).

\section{1. conteXto de LA INVEstigación}

Entre los estudios de pérdida de cosecha se encuentra el titulado "Evaluación de alternativas de manejo de poscosecha en hortalizas de hoja sobre las pérdidas a nivel minorista" (Ortiz y Grasso, p. 2016), el cual tuvo como objetivo "evaluar las pérdidas poscosecha en acelga, cebolla de verdeo, puerro, rúcula, radicheta y espinaca", donde se implementó una serie de actividades como la evaluación del efecto en el ambiente por medio del almacenamiento; los investigadores hacen énfasis en que hay pérdida de estos productos principalmente por falta de agua, lo que ocasiona marchitamiento; esto ocasiona pérdidas comerciales. Entre las variables de medidas se encontraron la pérdida de peso por descarte, pérdida o ganancia de pesos por agua y en porcentajes.

Del mismo modo (Ramírez, Jacobo, Ávila, \& Parra, 2006, pp. 45-10) han presentado el trabajo "Pérdidas de cosecha, eficiencia de producción y rentabilidad de huertos de manzano con diversos grados de tecnificación en Chihuahua, México"; en dicha investigación hicieron monitoreo durante el período 2001- 
2002, referente a la producción de manzana, donde se informa que la pérdida del fruto se origina por "efecto de las plagas, polinización deficiente y de frutos sanos"; allí se tomó como muestra 13 huertos de alta, media y baja tecnificación; para el análisis de los datos implementaron la prueba de medidas para estimar la rentabilidad y los sistemas de producción. Como resultado los investigadores resaltan diversas causas, entre las cuales enfatizan en la caída del fruto por el paso de la maquinaria y por la enfermedad llamada corazón mohoso, entre otros; igualmente manifiestan que las pérdidas en ese año de investigación se incrementaron en $21.8 \%$.

Es importante destacar el estudio presentado por (Lázaro, 2011) en Argentina, el cual analiza el instante recomendable de estado de madurez de este fruto en el momento de hacer la cosecha; como metodología empleada se utilizó un dispositivo espectroscópico Vis/ NIR, cuyo objetivo fue la clasificación del fruto en relación con la fecha establecida para la cosecha, destacando como fases de la investigación la firmeza, el color y la cantidad de sólidos del fruto. Esta investigación arroja como resultado que para obtener cirvelas en estado fresco se debe dar a conocer los valores de índice y destacar el estado de madurez de la cirvela; las cirvelas que presenten una madurez fenomenal serán utilizadas en procesos agroindustriales.

Por otra parte, (Sanchez, 2015) en un estudio determina la temperatura óptima de proceso con el objeto de minimizar la influencia de la temperatura de diferentes variedades de cirvela en la aparición de daños mecánicos originados en el procesado del producto y en las etapas post-cosecha; para realizar esta investigación hacen una simulación de almacenamiento de trasporte, implementando una cámara frigorífica por un lapso de tres días, haciendo un test (TVC) para mostrar el comportamiento del fruto en los supermercados durante cinco días. También hicieron la simulación de transporte mediante el almacenamiento en una cámara frigorífica a $4{ }^{\circ} \mathrm{C}$ durante tres días, estimando esta duración como un tiempo medio de transporte. Asimismo, se ha llevado a cabo un Test de Vida Comercial (TVC) de 5 días a $20^{\circ} \mathrm{C}$ simulando de esta forma el comportamiento que pudieran tener las cirvelas en el lineal del supermercado.
En Colombia se encuentran estudios de los cuales se resalta el presentado por (Rozo, Álvarez, \& Reyes, 2015), en el que hacen un estudio de comportamiento de poscosecha de la cirvela en diversos estados de madurez, implementando el etileno para la aceleración en aras de mostrar una mejor forma organoléptica. Para esta investigación se toman al azar cuatro momentos de madurez de fruto de cirvela y se controlan durante un período de tres días, analizando un total de 400 frutos, examinando el color, tasa respiratoria, peso y acidez, entre otros aspectos; como resultando exponen que el uso de etileno es favorable para realizar estados de madurez de estos frutos.

Para (Orjuela, 2016b), en un estudio efectuado en Nuevo Colón en el cual se buscó determinar la influencia de algunas variables climáticas y características del suelo en el desarrollo del fruto de cirvela y su calidad en cosecha, mediante la temperatura base del período fenológico comprendido entre cuaje de fruto y fruto en cosecha de la cirvela, tomando en total ochenta árboles, los cuales se manejaron de diez árboles por localidad, los resultados indican que las condiciones climáticas tienen gran influencia en el crecimiento y desarrollo del fruto de la cirvela, observándose cambios en cuanto a peso y tamaño de una cosecha a otra en las cuatro localidades estudiadas. Esto indica que los frutos que se producen a una altitud mayor $y$ menor (localidades 1 y 4) alcanzan un tamaño y peso mayores que los producidos a altitudes medias (localidades 2 y 3 ).

En cuanto a los estudios de cirvela, se encuentra el presentado por (Coronado, \& Camacho, 2007) cuyo objetivo es la evaluación de calidad de cirvela bajo tres condiciones de almacenamiento y refrigerado, mediante una análisis de varianza y comparación de medida implementando la prueba de ducan con un nivel de confianza de 5\%; los resultados de esta investigación permitirán a los cultivadores y comercializadores implementar un sistema de almacenamiento y refrigerado teniendo en cuenta las condiciones del ambiente, lo que permitirá mantener la calidad del producto y mejorar la comercialización, as de cuatro a cinco semanas.

Por su parte, (Gómez, \& Flórez, 2015) hacen énfasis en el estudio de cuatro cultivadores de 
cirvela en Japón, con el objeto de estudiar el efecto delatemperaturaenelalmacenamiento del fruto; el estudio comienza seleccionando los cultivadores de cirvela de piel roja y amarilla, posteriormente los trasladaron a laboratorios en un tiempo de dos horas para iniciar el procesos de preenfriamiento; como resultado los investigadores demuestran que el tiempo de traslado del producto generó reducción en la firmeza, causando un efecto negativo sobre el consumo.

\subsection{REVISIÓN DE LITERATURA}

\section{PRODUCCIÓN DE CIRUELA}

La producción de este fruto en Colombia ha presentado un crecimiento importante en la última década, situación originada por diversas causas entre las cuales se tiene la necesidad de consumir alimentos saludables y orgánicos que ayuden a mejorar la calidad de vida de los consumidores; además se ve reflejada la inclusión de nueva tecnología que ha permitido que los agricultores presenten diversificación del producto. Se presenta un incremento de $55 \%$ en el territorio nacional, destacando al departamento de Boyacá como el departamento que registra $74 \%$ de la comercialización del producto (Asohorfrucol, 2018).

Los municipios que tienen mayor producción de cirvela en el departamento de Boyacá son Nuevo Colón, con un rendimiento de 19 ton/ $\mathrm{Ha}$, lo que representa $40 \%$ del total producido; seguido por Tibaná y Jenesano, que producen 17 ton/Ha, y entre los dos suman 30\% del total producido; sigue Sotaquirá, con un rendimiento de 13 ton/ha y representa $13 \%$ del total producido; luego Tuta, con 10 ton/Ha y registra $10 \%$ de la producción total y los otros municipios registran el $7 \%$ restante con un rendimiento promedio de 8 ton/ha (Asohofrucol, 2018). Las unidades productivas de caducifolios en el departamento de Boyacá reúnen las siguientes características: área cultivada por productor, en promedio $3,72 \mathrm{ha}$, altas pendientes, $18,29 \%$ en $62 \%$ de las fincas, con mínimo 0,48 ha de la finca dedicada al cultivo de cirvela, 133 árboles/finca, edad promedio de las plantas, 7,79 años, rendimiento de 27,47 ton/ha.

Según un estudio hecho por Miranda (2013, p. 155), en un análisis preliminar de los sistemas productivos de caducifolios en este departamento se identifican dos problemas fundamentales: uno es la baja productividad de los huertos y el uso de variedades convencionales; el otro tiene que ver con la baja rentabilidad de los productores por la alta intermediación en la venta de los productos finales. La importancia de Boyacá en la explotación de cultivos de caducifolios se fundamenta en avances en el modelo de manejo empresarial, introducción de nuevas variedades, innovaciones tecnológicas, infraestructura de riego, trabajo asociativo, incremento del área cosechada, planeación de la producción acorde con el mercado, y consolidación de la cadena productiva. Se destaca como práctica fundamental la programación de las cosechas, la cual permite oferta de caducifolios en períodos de escasez con mejores precios de mercado (Puentes, Prieto, \& Caro, 2019). Miranda y Fischer anotan que en la actualidad las zonas de producción de caducifolios están dispersas por diferentes corregiones del país, con diversidad de cultivadores en su mayoría de procedencia desconocida, con niveles de uso de tecnología variables entre las regiones y entre las mismas fincas. Los sistemas de producción, de igual manera, presentan variaciones y niveles de desarrollo contrastantes.

\section{EVALUACIÓN DE PÉRDIDA DE COSECHA}

Entre los conceptos analizados se encuentran los mencionados en la metodología de evaluación de cadenas agroalimentarias (MECA), donde se indica que este fenómeno se origina por la disminución de la cantidad y calidad alimentaria desde el inicio del proceso de producción de la cosecha hasta el consumidor final; igualmente se mencionan entre las causas de las pérdidas la generación de impactos negativos en la sostenibilidad de las cadenas agroalimentarias debido al decrecimiento económico, la inversión y la reducción de la calidad y disposición de los alimentos, entre otros aspectos.

Por esta razón se quiere hacer la evaluación, para la cual menciona la (Organización de las Naciones Unidad para la Alimentación y la Agricultura, FAO), s. f.), los siguientes pasos a fin de conseguir el objetivo.

Hacer una encuesta general antes de dar inicio al proyecto de reducción de pérdidas 
de cosecha, posteriormente manejar una encuesta piloto aplicando una metodología cuantitativa con el fin de establecer las pérdidas y obtener datos de las causas.

Hacer ensayos de campo, para comparar las pérdidas que se presentan y utilizar estrategias para mejorarlas; se debe tener en cuenta tres tipos de ensayos: ensayo de la eficiencia del equipo, ensayo de simulación de almacenamiento en estaciones de investigación, y ensayos en las granjas.

Posteriormente se hace una validación de la encuesta y los ensayos de validez de la encuesta y los trabajos de ensayos.

Para la evaluación de las pérdidas se tendrán en cuenta los datos recopilados en campo, tomándose como base en la producción y a variables de pérdida como el número de frutos totales por árbol, frutos sobremaduros, frutos caídos por accidente, frutos aptos no cosechados, frutos desechados por daño, metodología similar a Arauz y Mora (1983). Algunas de las causas que provocan las pérdidas en los cultivos de cirvela son la inadecuada manipulación, la incidencia de plagas y enfermedades, el picoteo de aves, la sobremadurez de la fruta; aspectos que llevaron a encontrar frutos con daños físicos, mecánicos y fisiológicos.

\section{2. metodología}

Este trabajo se desarrolló a través de la metodología acción - participación, en donde los productores de cirvela Horvin fueron los actores más importantes del proyecto, considerándolos la fuente primaria para poder recolectar la información; fueron de gran ayuda para el buen desarrollo de los planes requeridos en las unidades productivas, aspecto fundamental para la toma de decisiones y la obtención de los mejores resultados.

El método utilizado fue el estudio de caso, que consistió en utilizar los cinco ejemplos reales de la producción de cirvela con el objeto de profundizar en el conocimiento del tema analizado, así fue posible extraer una serie de datos aplicables al proceso de planificación.
El objetivo del estudio de caso fue responder a las preguntas acerca de cómo se realiza la producción, qué insumos utilizaron, cuáles fueron las épocas de las labores, qué variables trabajaron para desplazar las cosechas.

El estudio de caso permitió incluir el análisis de documentos, de datos estadísticos o datos de ejecución, pero lo que se implicó y destacó especialmente fue la observación directa del fenómeno estudiado y las entrevistas a las personas directamente implicadas. El trabajo se desarrolló mediante el estudio de caso, que definió cómo estudiar el caso de 5 productores de cirvela de una zona específica, con características homogéneas de clima y suelos, como sistema de producción de cirvela y ámbito social, se investigará cómo y por qué se presenta la producción en las mismas épocas del año.

En cada una de las fincas se utilizó la metodología de planificación y producción de cultivos (Alba, 2012), en donde se tuvieron en cuenta aspectos como: selección del cultivo, temporada, clima, mercado, mano de obra, costos, plagas y enfermedades, asociación con otros cultivos, fertilización y dentro de estos el cómo: 1. dibujando el mapa de las fincas; 2. estableciendo las características de la producción de cirvela (enfoque de sistemas); 3 . descripción botánica de la planta y los estados fenológicos; 4. elaboración de diagramas de producción; insumos, cosechas y mano de obra; 5 . elaboración de registros de producción.

\subsection{POBLACIÓN Y MUESTRA}

Las fincas productoras de cirvela Horvin son 5 de la vereda Río de piedras, del municipio de Tuta, dadas las características homogéneas que presenta la zona, el sistema de producción, la experiencia de los productores y los niveles de conocimiento de los pequeños productores.

\subsection{RECOLECCIÓN DE LA INFORMACIÓN}

Para recolectar la información se diseñó una ficha técnica, con la descripción de los estados fenológicos de la cirvela, las labores culturales que se hacen en un cultivo de cosecha a cosecha, el estado de producción de cirvela Horvin en la vereda, los rendimientos por cultivo; esta ficha se diligenció con cada productor en la finca, además se hizo observación directa 
en las fincas $y$ entrevistas al personal que labora y a cada uno de los productores; al tener la mayor parte de la información se hizo una reunión con los productores, en donde se presentó un esquema del cultivo y de cada una de las prácticas que realizaron, para validar la información.

\subsection{PROCESAMIENTO DE LA INFORMACIÓN}

Se hizo mediante el análisis de las encuestas y observaciones que se desarrollaron sobre la planeación y comercialización de cirvela Horvin en la vereda, donde por medio de gráficos se tabulará y se analizará la información recolectada para sacar datos estadísticos con mayor exactitud; así se determinaron las causas por las cuales se presenta una sobreproducción en las mismas épocas del año; se verificó el tiempo, la duración de la cosecha, los indicadores de rendimientos de mano de obra, las prácticas culturales y la representación del ciclo productivo de cosecha a cosecha en cada una de las fincas.

\subsection{ANÁLISIS DE LA INFORMACIÓN}

Después de la tabulación de las encuestas y del análisis de la producción en cada una de las unidades productivas, se definieron las variables que se tuvieron en cuenta para adelantar o retrasar cosechas, aspecto que se trabajó con ayuda de información secundaria, así como la problemática más relevantes que tienen los productores para la planeación y comercialización de la cirvela Horvin; con este trabajo se lograron elementos necesarios para la elaboración de los respectivos planes de producción, cosecha, cronograma de actividades, cronograma de insumos y de mano de obra.

\section{RESULTADOS}

Planificación de CULTIVARES de cirvela. Se desarrolló cada uno de los pasos de la planificación agropecuaria, con una explicación detallada de un caso práctico sobre el cultivo de cirvela en el municipio de Tuta, Boyacá.

1. Identificación. Sistema de producción. Cultivos de cirvela, variedad Horvin.
2. Contextualización: La planificación se hará en el municipio de Tuta, Boyacá, que pertenece a la provincia centro del departamento de Boyacá; está distribuido en veredas: Cabecera, Hacienda, Río de Piedras, Resguardo, Agua Blanca, Hato, Alisal, San Nicolás y Leonera. El número de habitantes en la parte urbana es 3.912 y en la parte rural 8.789. Las fincas se encuentran situadas en la vereda Río de Piedras del municipio de Tuta, el cual cuenta con una población de 801 habitantes; están localizadas sobre el eje central de la vía Paipa - Tunja, kilómetro 17. Su economía está enfocada principalmente en la ganadería y la producción de caducifolios como principal fuente de ingresos.

3. Misión de la empresa Ciruelas Coronado. "Producir y comercializar cirvela Horvin de excelente calidad, atendiendo las exigencias del mercado, con oportunidad, continuidad y cumplimiento".

Se reunieron los conocedores de la empresa o negocio, se presentaron 5 preguntas, se nombró un moderador, que fue haciendo cada pregunta y escribiendo las respuestas de cada uno de los asistentes; se analizaron las respuestas y se organizaron los elementos que al final se reunieron y así se redactó la misión.

Se volvió a formular la misión, incorporando los elementos que faltaron y concretando un escrito corto, claro y preciso, que reflejó el quehacer de la empresa.

Culminando lo anterior se formularon los Objetivos de la empresa Ciruelas Coronado:

- Producir cirvela Horvin constantemente y satisfacer la demanda del mercado regional.

- Planificar las cosechas en cinco fincas seleccionadas.

- Elaborar los planes financieros de las fincas.

Visión. Para la formulación de la visión se utilizaron iguales metodologías que para la misión, pero se hicieron preguntas a futuro: ¿cómo ven ustedes la producción de cirvela dentro de diez años? ¿̇cuáles serán las exigencias de los futuros consumidores de cirvela? ¿A dónde más se pretende llegar 
con la cirvela Horvine, ¿Qué tecnologías se implementarán para la producción de cirvela en los próximos diez años?

\section{Visión de la empresa Ciruelas Coronado.} "Producir cirvela Horvin de excelente calidad, con cumplimiento de certificación BPA y con comercialización de fruta durante todo el año".

Ya elaborados los tres pasos anteriores: selección del tema, contextualización y misión, visión objetivos y valores, se procedió a la elaboración del diagnóstico, se describieron los aspectos que coadyuvaron al logro de resultados eficientes y eficaces sobre el tema por tratar. Se recolectó y sistematizó la información como se describe a continuación. En la elaboración del diagnóstico vale la pena resaltar el enfoque de sistemas, el cual nace con la teoría general de los sistemas según Von Bertalanfly (1998), se logró estructurar con enfoque sistémico la unidad agrícola con detalle y precisiones en los tres elementos, como se observa en la Tabla 1.

Luego se pasó al diseño de las estrategias, se identificaron cuáles son los aspectos

Tabla 1. El enfoque de sistemas, "sistema de producción de la cirvela en el municipio de Tuta, Boyacá".

\begin{tabular}{|c|c|c|}
\hline Entradas & Procesos & Salidas \\
\hline $\begin{array}{l}\text { Aspectos climáticos } \\
\text { Clima: zona templada, es muy } \\
\text { resistente al frío, hasta } 400 \text { horas } \\
\text { frío. } \\
\text { - Temperatura: } 18 \text { oC a } 25 \text { OC } \\
\text { - Horas frío: } 400-500 \text { hora/año } \\
\text { - Agua: } 1 \text { - } 10 \text { L/ha } \\
\text { - Luz: } 90 \text { horas/ mes } \\
\text { - Pluviosidad: } 600 \text { y } 1.300 \text { mm } \\
\text { - Humedad: } 90 \% \\
\text { Suelo: aguanta bien la caliza, } \\
\text { la humedad y los terrenos } \\
\text { compactados. Excepto suelos } \\
\text { arenosos. }\end{array}$ & $\begin{array}{l}\text { Seleccionar la zona para } \\
\text { los cultivos de cirvela. } \\
\text { Analizar las condiciones } \\
\text { climáticas y las exigencias } \\
\text { de la especie. }\end{array}$ & $\begin{array}{l}\text { Condiciones aptas para el } \\
\text { cultivo. }\end{array}$ \\
\hline $\begin{array}{l}\text { Aspecto tecnológico / produción } \\
\text { - Conocimiento, terreno, mano } \\
\text { de obra, tractor, planos, } \\
\text { metros, estacas, cuerda, } \\
\text { gallinaza, cal dolomita, boro, } \\
\text { fósforo, potasio. } \\
\text { - } 625 \text { plantas por hectárea. } \\
\text { - Sistema de riego: agua, } \\
\text { manguera, medidor, llave de } \\
\text { paso, goteros, filtros, bomba de } \\
\text { motor. } \\
\text { - fertilizantes foliares, } \\
\text { - Herramientas: tijeras de poda, } \\
\text { rastrillo. } \\
\text { - Fungicidas } \\
\text { - canastillas, sala de } \\
\text { poscosecha. }\end{array}$ & $\begin{array}{l}\text { Planear la producción. } \\
\text { Preparar el terreno: arar, } \\
\text { rastrillar, trazar, ahoyar, } \\
\text { fertilizar. } \\
\text { Conseguir el material } \\
\text { vegetativo, plántulas. } \\
\text { Sembrar } \\
\text { Implementar sistema de } \\
\text { riego } \\
\text { Fertilizar Podar Fumigar } \\
\text { Cosechar } \\
\text { Seleccionar producción } \\
\text { Clasificar Almacenar. }\end{array}$ & $\begin{array}{l}\text { Planeación proceso de } \\
\text { producción } \\
\text { Terreno disponible, preparado } \\
\text { para siembra } \\
\text { Plantas en sitio definitivo } \\
\text { Cultivo instalado } \\
\text { Riego instalado y agua } \\
\text { disponible para el cultivo } \\
\text { Plantas fertilizadas } \\
\text { Plantas podadas } \\
\text { Plantas listas para reposo y } \\
\text { floración } \\
\text { Fruto recolectado y } \\
\text { empacado } \\
\text { Producto en espera para ser } \\
\text { distribuido. }\end{array}$ \\
\hline
\end{tabular}


Tabla 1. Continuación

\begin{tabular}{|c|c|c|}
\hline Entradas & Procesos & Salidas \\
\hline $\begin{array}{l}\text { Aspecto de comercialización } \\
\text { - Exigencias de los consumidores, } \\
\text { información, conocimiento, } \\
\text { software, personal. } \\
\text { - } \quad \text { Precios del mercado, costos } \\
\text { de producción, demanda del } \\
\text { producto, lugares de compra. } \\
\text { - Conocimiento del tema, } \\
\text { contactos, clientes, productos } \\
\text { terminados de calidad. } \\
\text { Producto, pedido del } \\
\text { producto, vehículo, } \\
\text { conductores, canales de } \\
\text { distribución. }\end{array}$ & $\begin{array}{l}\text { Investigar el mercado } \\
\text { Asignar precios } \\
\text { Negociar } \\
\text { Transportar y distribuir }\end{array}$ & $\begin{array}{l}\text { Características de la } \\
\text { población, gastos del cliente, } \\
\text { características del producto, } \\
\text { estudio a la competencia, } \\
\text { identificación del mercado } \\
\text { objetivo. } \\
\text { Asignación de precios. } \\
\text { Acuerdos comerciales y venta } \\
\text { del producto y pedidos. } \\
\text { Ingresos por venta, producto } \\
\text { entregado. }\end{array}$ \\
\hline $\begin{array}{l}\text { Aspecto económico y financiero } \\
\text { - Información, recursos, } \\
\text { computadores, papelería, } \\
\text { servicio, software, capital. } \\
\text { - Utilidad de la producción. }\end{array}$ & $\begin{array}{l}\text { Diseñar formatos de } \\
\text { inventarios, } \\
\text { Manejar software, crear } \\
\text { hojas de cálculo } \\
\text { Elaborar nómina, } \\
\text { Tributar a las empresas y al } \\
\text { Estado. }\end{array}$ & $\begin{array}{l}\text { Costos de producción, } \\
\text { balance general, estado de } \\
\text { ganancias y pérdidas. } \\
\text { Aportación al PIB. }\end{array}$ \\
\hline $\begin{array}{l}\text { Aspecto social } \\
\text { - Mano de obra, cargos, } \\
\text { caracterización de la } \\
\text { población y ubicación. }\end{array}$ & $\begin{array}{l}\text { Generar y contribuir al } \\
\text { aumento del empleo en el } \\
\text { sector rural. }\end{array}$ & $\begin{array}{l}\text { Generación de empleo, } \\
\text { contribución al mejoramiento } \\
\text { de la calidad de vida de los } \\
\text { habitantes. }\end{array}$ \\
\hline $\begin{array}{l}\text { Aspectos administrativos } \\
\text { - Información de la producción, } \\
\text { de las actividades del cultivo y } \\
\text { del mercado. } \\
\text { - Información de actividades } \\
\text { de compra, comercialización, } \\
\text { información sobre registro } \\
\text { personal (puntualidad, } \\
\text { rendimiento, conducta, } \\
\text { actitud, desempeño), } \\
\text { información de recursos } \\
\text { financieros, información de } \\
\text { rendimiento de producción. } \\
\text { - Conocimiento de tareas, } \\
\text { talento humano. } \\
\text { Talento humano, procesos } \\
\text { técnicos y administrativos, } \\
\text { investigación. }\end{array}$ & $\begin{array}{l}\text { Planear } \\
\text { Contratar mano de obra } \\
\text { Controlar y medir }\end{array}$ & $\begin{array}{l}\text { Toma de decisiones, } \\
\text { planificación de la } \\
\text { producción, cronograma } \\
\text { de actividades del cultivo, } \\
\text { estudio del mercado. } \\
\text { Evaluación del personal, } \\
\text { manual de procedimientos, } \\
\text { estados de resultados, manejo } \\
\text { de inventarios, medición de } \\
\text { indicadores de producción, } \\
\text { estructura organizacional. }\end{array}$ \\
\hline
\end{tabular}


Tabla 1. Continuación

\begin{tabular}{|c|c|c|}
\hline Entradas & Procesos & Salidas \\
\hline $\begin{array}{l}\text { Aspectos ambientales } \\
\text { Insumos, productos químicos, } \\
\text { orgánicos y biológicos. } \\
\text { - Residuos de cosecha } \\
\text { - Fuentes hídricas } \\
\text { - Suelo desgastado }\end{array}$ & $\begin{array}{l}\text { Recolectar. } \\
\text { Transformar residuos }\end{array}$ & $\begin{array}{l}\text { Contaminación al medio } \\
\text { ambiente } \\
\text { Humus } \\
\text { Uso racional del agua en la } \\
\text { producción. } \\
\text { Suelo preparado para la } \\
\text { plantación. }\end{array}$ \\
\hline $\begin{array}{l}\text { Talento humano } \\
\text { - Mano de obra } \\
\text { - Documentos } \\
\text { - Computador }\end{array}$ & $\begin{array}{l}\text { Describir cargos } \\
\text { Asignar funciones } \\
\text { Convocar el personal } \\
\text { Selección del personal } \\
\text { Capacitar el personal } \\
\text { Contratar el personal }\end{array}$ & $\begin{array}{l}\text { Manual de funciones } \\
\text { Base de datos de hojas de } \\
\text { vida } \\
\text { Capacitar al personal }\end{array}$ \\
\hline
\end{tabular}

Fuente: Coronado (2015).

positivos y negativos con que cuenta el sistema. La evaluación se hizo de manera interna (debilidades y fortalezas) y externa (oportunidades y amenazas), para así aprovechar las diferentes herramientas e identificar acciones o estrategias y además definir los objetivos que realmente el sistema debe cumplir.

\section{Estructura de la matriz DOFA y formulación de la estrategia \\ La matriz DOFA es un procedimiento de} gran utilidad para afianzar la comprensión de los conceptos previamente analizados, sobre todo en lo referente a la definición de estrategias por llevar a cabo. Mediante un ejemplo que se desarrolló en la vereda Río de Piedras, del municipio de Tuta, se da a conocer la utilidad de esta herramienta; ver la Tabla 2 y los demás aspectos del proceso de planificación agropecuaria.

En el análisis de la matriz DOFA se puede deducir que a pesar de que los cultivos son pequeños, para los productores representan una inversión importante que cada seis meses genera ingresos; de ahí que se planteen estrategias como la FA, planificación de los cultivos para desplazar la producción y lograr mejores precios en el mercado; además se observa la falta de apoyo por las entidades del Estado en cuanto a investigación, manejo administrativo y asistencia técnica.

5. Selección de la estrategia. Estrategia: FA. Diseñar los planes de fertilización, podas, labores en donde sea posible acelerar o atrasar el proceso productivo de la cirvela cuando la oferta lo requiera. En una de las etapas del proceso de planificación estratégica del agronegocio (Guerra, 1992) se hizo el análisis; se priorizaron y valoraron las fortalezas y las debilidades de este sistema en la vereda Río de Piedras del municipio de Tuta, así como el análisis del entorno. Con el análisis anterior se elaboró un consolidado de los aspectos internos y externos que se encuentran en el manejo de estos sistemas productivos.

\section{Elaboración de planes de acción}

Plan técnico. Para el desarrollo del plan técnico se inició con un bosquejo sobre el ciclo productivo de la cirvela, en donde el profesional debe conocer qué pasa en cada estado fenológico y cómo se comporta en la región, como se muestra en la Figura 1. 
Tabla 2. Representación de la matriz DOFA para el cultivo de cirvela en la vereda Río de Piedras, municipio de Tuta, Boyacá.

\begin{tabular}{|c|c|c|}
\hline Análisis externo & $\begin{array}{l}\text { Fortalezas } \\
\text { Cercanía con centros de acopio y } \\
\text { de consumo. } \\
\text { Fruta fresca en varios meses del } \\
\text { año. } \\
\text { Condiciones climáticas favorables. } \\
\text { Experiencia de los productores. } \\
\text { Fruta de buena calidad. }\end{array}$ & $\begin{array}{l}\text { Debilidades } \\
\text { Deficiente manejo poscosecha. } \\
\text { No hay organización de los } \\
\text { productores. } \\
\text { La existencia de bajos precios. } \\
\text { Deficiencia en el manejo } \\
\text { administrativo. } \\
\text { El manejo técnico es bajo. } \\
\text { Carencia de investigación. } \\
\text { Incidencia de enfermedades en } \\
\text { el cultivo }\end{array}$ \\
\hline $\begin{array}{l}\text { Oportunidades } \\
\text { Facilidad en acceso a } \\
\text { créditos. } \\
\text { Existen programas de } \\
\text { apoyo y financiamiento } \\
\text { a pequeños } \\
\text { productores. } \\
\text { Generación de empleo } \\
\text { e ingresos para las } \\
\text { personas de la región. }\end{array}$ & $\begin{array}{l}\text { Estrategia FO } \\
\text { Trabajar los cultivos con } \\
\text { cosechas forzadas para permitir } \\
\text { la disponibilidad de fruta fresca } \\
\text { todo el año, y así mantener la } \\
\text { generación de empleo, lo cual } \\
\text { lleva al mejoramiento de los } \\
\text { niveles de vida de los empleados. }\end{array}$ & $\begin{array}{l}\text { Investigar en el almacenamiento } \\
\text { en frío de la fruta, para prolongar } \\
\text { la vida útil de la ciruela, variedad } \\
\text { Horvin, dada la característica de } \\
\text { ser altamente perecedera. }\end{array}$ \\
\hline $\begin{array}{l}\text { Amenazas } \\
\text { Las excesivas } \\
\text { importaciones. } \\
\text { Alta competencia en el } \\
\text { mercado interno. } \\
\text { Compra de insumos } \\
\text { sobre el valor del dólar } \\
\text { y el euro. } \\
\text { El sistema de los } \\
\text { caducifolios no se } \\
\text { encuentra como } \\
\text { renglón prioritario. }\end{array}$ & $\begin{array}{l}\text { Estrategia FA } \\
\text { Diseñar los planes de fertilización, } \\
\text { podas, labores en donde sea } \\
\text { posible acelerar o atrasar el } \\
\text { proceso productivo de la cirvela } \\
\text { cuando la oferta lo requiera. }\end{array}$ & $\begin{array}{l}\text { Estrategia DA } \\
\text { Al realizar una administración } \\
\text { correcta de la finca se disminuirán } \\
\text { las pérdidas de producto y se } \\
\text { hará uso eficiente de los recursos. }\end{array}$ \\
\hline
\end{tabular}

Fuente: Coronado (2015). 
Figura 1. Desarrollo del cultivo cirvela Horvin en los primeros 10 años

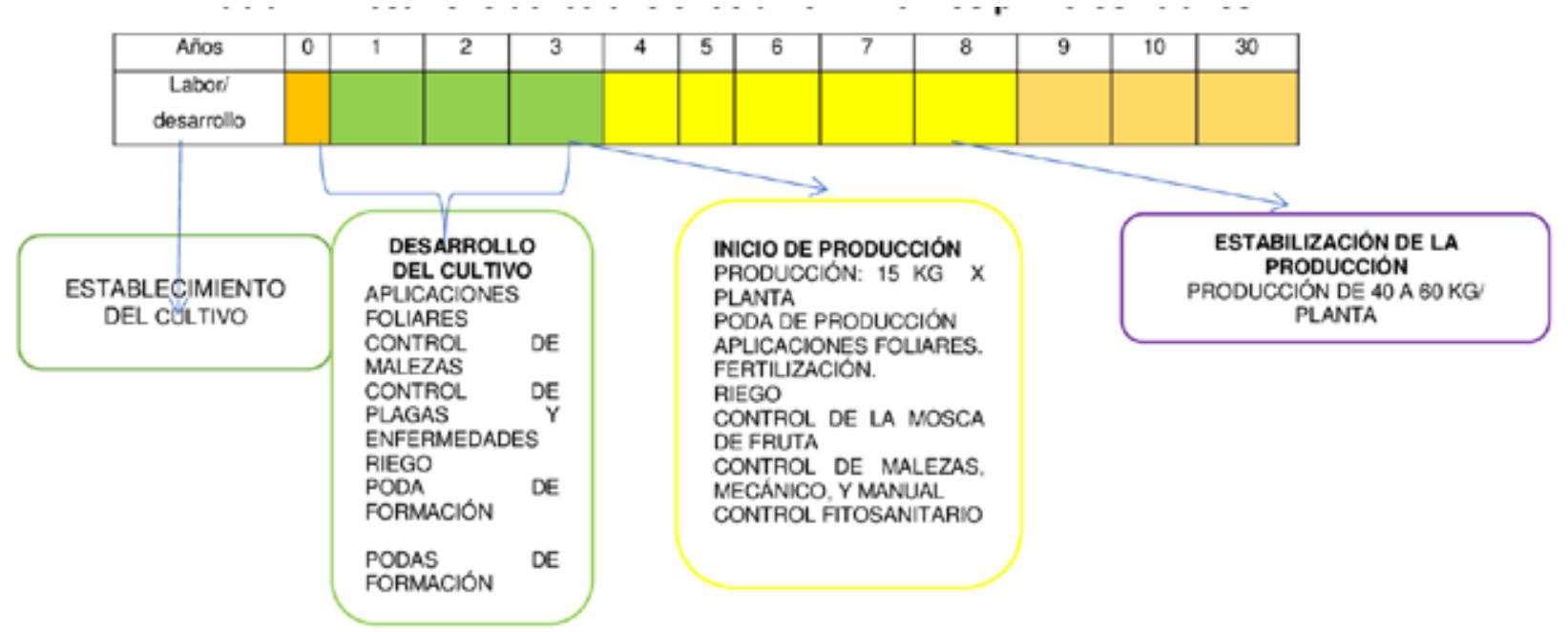

Fuente: Coronado (2015).

Igualmente se realiza una caracterización de cinco cultivos, destacando algunos aspectos de cada finca encuestada, como se evidencia en la Tabla 3.

Los cultivos de ciruela Horvin estudiados son pequeños, cuentan con el establecimiento de 100 a 450 plantas, manejan unas distancias adecuadas para la producción con rendimiento promedio por planta de 40 $\mathrm{kg}$, lo cual lleva a que haya una excelente producción, desde $4.000 \mathrm{~kg}$ hasta 1.600 $\mathrm{kg}$, aspecto que permite manejar una comercialización conjunta y hacer una planificación de cosechas, para tratar de tener producto fresco durante un buen número de semanas al año y así favorecer a los pequeños productores con el incremento de los ingresos, dados los buenos precios del mercado, sobre todo en épocas de escasez de la fruta en otras regiones del país.

Prácticas culturales En la producción de cirvela se requieren diferentes labores culturales que son necesarias en cada uno de los estados fenológicos, así como la aplicación de insumos con su dosis respectiva con el fin de tener una excelente cosecha; estas se presentan en la Tabla 4.

En el proceso de producción de la cirvela y para llevar a cabo la planeación de cosechas, es importante elaborar los cuadros con requerimientos de insumos. En los siguientes cuadros se muestra cada uno de los insumos requeridos en la producción; cabe resaltar que la fertilización se realiza dos veces en el año; los productores de cirvela en Tuta la hacen en el estado fenológico de la floración para estimular la yema, porque ayuda al cuajamiento de la flor; también hacen controles fitosanitarios para plagas y enfermedades, con aplicaciones de fungicidas, que además llevan fertilizantes foliares; la utilización de bactericidas e insumos de engruese; también hacen control de arvenses, estas aplicaciones las hacen teniendo en cuenta las recomendaciones de un ingeniero agrónomo (Tabla 5).

En la Tabla 6 se puede apreciar la cantidad de insumos requeridos para la producción de cirvela, encontrando que en la finca en que más insumos se necesitan es la finca cuatro; debido al número de plantas y a que el propietario cuenta con recursos para hacer las respectivas aplicaciones, se aplicarán 2 $\mathrm{kg}$ de abono por planta, esto hace que las plantas tengan un mejor desarrollo $y$, por ende, mejor rendimiento en la producción; los demás productores harán aplicaciones de un kilogramo por planta; en todos los casos las aplicaciones son recomendadas por un ingeniero agrónomo.

Requerimiento de mano de obra: el cultivo de cirvela tiene seis importantes labores culturales, las cuales son la base para un buen desarrollo de este. A continuación se mostrará el número de jornales que se requieren para una finca con las labores culturales; en el plan técnico se llama requerimientos de mano de obra (Figura 2).

En cuanto al requerimiento de mano de obra presentado, se puede analizar que todas las fincas hacen las mismas labores culturales, de 
Tabla 3. Características de cinco cultivos de cirvela en el municipio de Tuta.

\begin{tabular}{|c|c|c|c|c|c|}
\hline $\begin{array}{l}\text { Nombre de } \\
\text { la finca }\end{array}$ & Villa Diego (1) & El Cerezo (2) & (3) Domo & $\begin{array}{l}\text { La Esperanza } \\
\text { (4) }\end{array}$ & El Primor (5) \\
\hline $\begin{array}{l}\text { \# De } \\
\text { Fanegadas }\end{array}$ & 3 & 1 & 2 & 4 & 3 \\
\hline $\begin{array}{l}\text { Número de } \\
\text { plantas }\end{array}$ & 250 & 200 & 450 & 380 & 100 \\
\hline $\begin{array}{l}\text { Distancia de } \\
\text { siembra }\end{array}$ & $4 \times 4$ & $3,5 \times 3,5$ & $3,5 \times 3,5$ & $4 \times 4$ & $4 \times 4$ \\
\hline $\begin{array}{l}\text { Rendimiento } \\
\text { por planta }\end{array}$ & $43 \mathrm{~kg}$ & $40 \mathrm{~kg}$ & $37 \mathrm{~kg}$ & $43 \mathrm{~kg}$ & $39 \mathrm{~kg}$ \\
\hline $\begin{array}{l}\text { Área del } \\
\text { cultivo }\end{array}$ & $1 / 2$ fanegada & $1 / 2$ fanegada & 1 fanegada & 1 fanegada & $1 / 2$ fanegada \\
\hline $\begin{array}{l}\text { Edad de los } \\
\text { cultivos }\end{array}$ & 5 años & 6 años & 8 años & 8 años & 5 años \\
\hline $\begin{array}{l}\text { Características } \\
\text { del suelo }\end{array}$ & $\begin{array}{l}\text { Franco } \\
\text { arcillosos }\end{array}$ & $\begin{array}{l}\text { Franco } \\
\text { arcillosos }\end{array}$ & $\begin{array}{l}\text { Franco } \\
\text { arenosos }\end{array}$ & $\begin{array}{l}\text { Franco } \\
\text { arcillosos }\end{array}$ & $\begin{array}{l}\text { Franco } \\
\text { arcillosos }\end{array}$ \\
\hline
\end{tabular}

Fuente: Coronado (2015).

Tabla 4. Descripción de las labores culturales y requerimientos de insumos de cosecha a cosecha

\begin{tabular}{|c|c|c|c|c|}
\hline E $\begin{array}{c}s \dagger a \\
\text { fenológico }\end{array}$ & $\begin{array}{c}\text { Duración } \\
\text { días }\end{array}$ & Labores culturales & Insumos requeridos & Dosis \\
\hline Reposo & 30 & $\begin{array}{l}\text { Aplicaciones para } \\
\text { llenado de yemas, } \\
\text { y maduración de } \\
\text { hojas. } \\
\text { Podas de } \\
\text { producción }\end{array}$ & $\begin{array}{l}\text { Sulfato de potasio, } \\
\text { mancozeb } \\
\text { podadoras }\end{array}$ & $\begin{array}{l}200 \operatorname{cc} x \\
20 L\end{array}$ \\
\hline Defoliación & 30 & $\begin{array}{l}\text { Se realiza una } \\
\text { aplicación para } \\
\text { la caída de las } \\
\text { hojas, también se } \\
\text { hace la poda de } \\
\text { producción. }\end{array}$ & $\begin{array}{l}\text { Sulfato de cobre, } \\
\text { sulfato de zinc, } \\
\text { nitrato de potasio. }\end{array}$ & $\begin{array}{l}100 \mathrm{cc} \times 20 \mathrm{~L} 50 \mathrm{cc} \times \\
20 \mathrm{~L} 100 \mathrm{cc} \times 20 \mathrm{~L}\end{array}$ \\
\hline Floración & 30 & $\begin{array}{l}\text { Se aplica } \\
\text { compensador de } \\
\text { frio, y una aplicación } \\
\text { de fósforo. }\end{array}$ & Dormex, fósforo & $100 \mathrm{cc} \times 20 \mathrm{~L}$ \\
\hline $\begin{array}{l}\text { Desarrollo del } \\
\text { fruto }\end{array}$ & 70 & $\begin{array}{l}\text { Se hacen diferentes } \\
\text { aplicaciones de } \\
\text { fertilizantes foliares y } \\
\text { controles de plagas y } \\
\text { enfermedades }\end{array}$ & $\begin{array}{l}\text { Fertilizantes que } \\
\text { contengan macro } \\
\text { y micronutrientes, } \\
\text { fungicidas, } \\
\text { insecticidas. }\end{array}$ & $\begin{array}{l}1.000 \mathrm{~g} \times \text { planta } 50 \\
\mathrm{cc} \times 20 \mathrm{~L}\end{array}$ \\
\hline Cosecha & 25 a 30 & $\begin{array}{l}\text { Se hace cada } \\
\text { semana y se } \\
\text { recolecta en estado } \\
\text { de madurez pintona. }\end{array}$ & Baldes y canastillas. & \\
\hline
\end{tabular}

Fuente: Coronado (2015). 
Tabla 5. Planeación de los requerimientos de insumos en las cinco fincas estudiadas

\begin{tabular}{|c|c|c|c|c|c|c|}
\hline Insumos & Unidad & $\begin{array}{l}\text { Cantidad } \\
\text { Fincal }\end{array}$ & $\begin{array}{c}\text { Cantidad } \\
\text { Finca } 2\end{array}$ & $\begin{array}{c}\text { Cantidad } \\
\text { Finca3 }\end{array}$ & $\begin{array}{l}\text { Cantid ad } \\
\text { Finca } 4\end{array}$ & $\begin{array}{l}\text { Cantidad } \\
\text { Finca } 5\end{array}$ \\
\hline $\begin{array}{l}\text { Número de } \\
\text { plantas }\end{array}$ & & 250 & 200 & 380 & 4.500 & 100 \\
\hline $10-30-10$ & bulto x $50 \mathrm{~kg}$ & 5 bultos & 4 bultos & 8 bultos & 9 bultos & 2 bultos \\
\hline $\begin{array}{l}\text { Abono } \\
\text { orgánico } \\
\text { (gallinaza) }\end{array}$ & bulto x $50 \mathrm{~kg}$ & 15 bultos & 20 bultos & 22 bultos & 45 bultos & 6 bultos \\
\hline $\begin{array}{l}\text { Elementos } \\
\text { menores } \\
\text { (Agrimins) }\end{array}$ & bulto x $50 \mathrm{~kg}$ & 2 bultos & 2 bultos & 3 bultos & 5 bultos & 1 bultos \\
\hline $\begin{array}{l}\text { Fertilizantes } \\
\text { foliares }\end{array}$ & L & $6 \mathrm{~L}$ & $5 \mathrm{~L}$ & $9 \mathrm{~L}$ & $12 \mathrm{~L}$ & $3 L$ \\
\hline $\begin{array}{l}\text { Fertilizantes } \\
\text { de } \\
\text { engruese }\end{array}$ & L & $3 \mathrm{~L}$ & $3 \mathrm{~L}$ & $6 \mathrm{~L}$ & $6 \mathrm{~L}$ & $2 \mathrm{~L}$ \\
\hline Insecticidas & & & & & & $1 \mathrm{~L}$ \\
\hline Lorban & $\mathrm{L}$ & $1 \mathrm{~L}$ & $1 \mathrm{~L}$ & $2 \mathrm{~L}$ & 1 & 2 \\
\hline \multicolumn{7}{|l|}{ Fungicidas } \\
\hline Ortocide & Bolsa $1 \mathrm{~kg}$ & $3 \mathrm{~kg}$ & $3 \mathrm{~kg}$ & $5 \mathrm{~kg}$ & $7 \mathrm{~kg}$ & $3 \mathrm{~kg}$ \\
\hline Antracol & Bolsa $1 \mathrm{~kg}$ & $3 \mathrm{~kg}$ & $3 \mathrm{~kg}$ & $6 \mathrm{~kg}$ & $6 \mathrm{~kg}$ & $2 \mathrm{~kg}$ \\
\hline \multicolumn{7}{|c|}{ Bactericidas } \\
\hline Escore & $250 \mathrm{cc}$ & 3 & 1 & 5 & 7 & 2 \\
\hline Herbicidas & Galón & 2 & 1 & 2 & 2 & 1 \\
\hline
\end{tabular}

Fuente: Coronado (2015).

Figura 2. Planeación de mano de obra

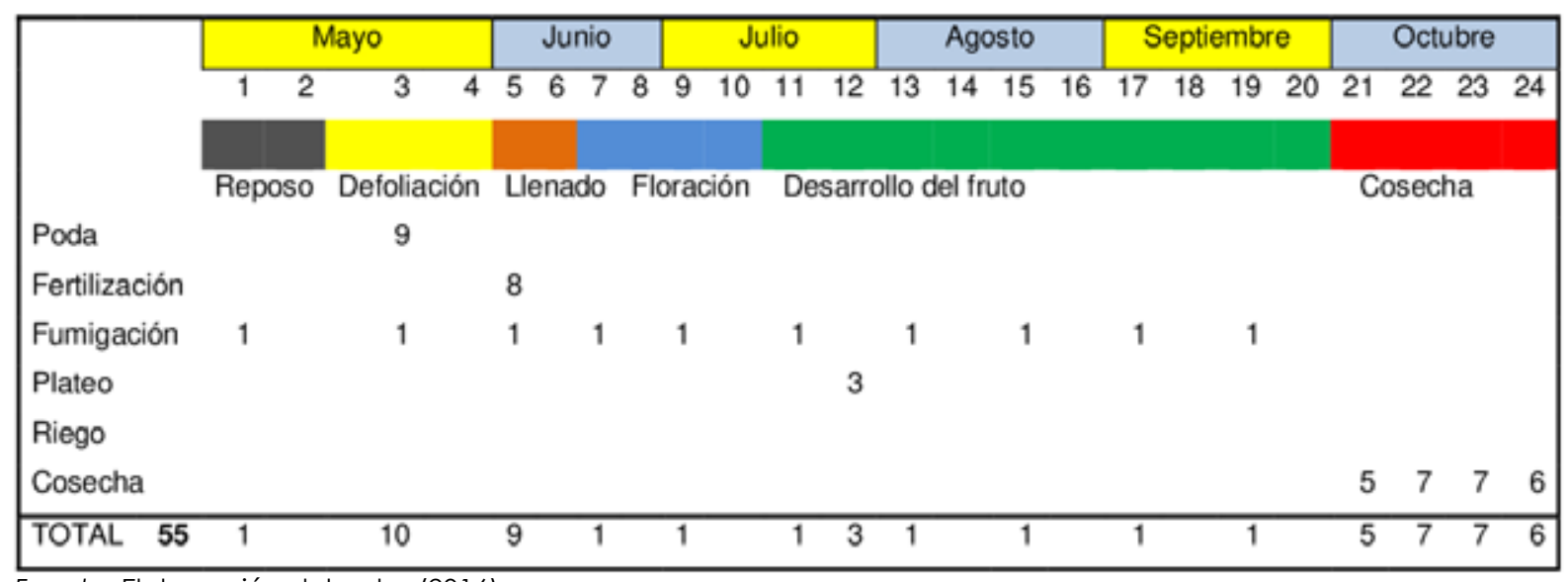

Fuente: Elaboración del autor (2016). 
Figura 3. Fenología del cultivo de cirvela, variedad Horvin

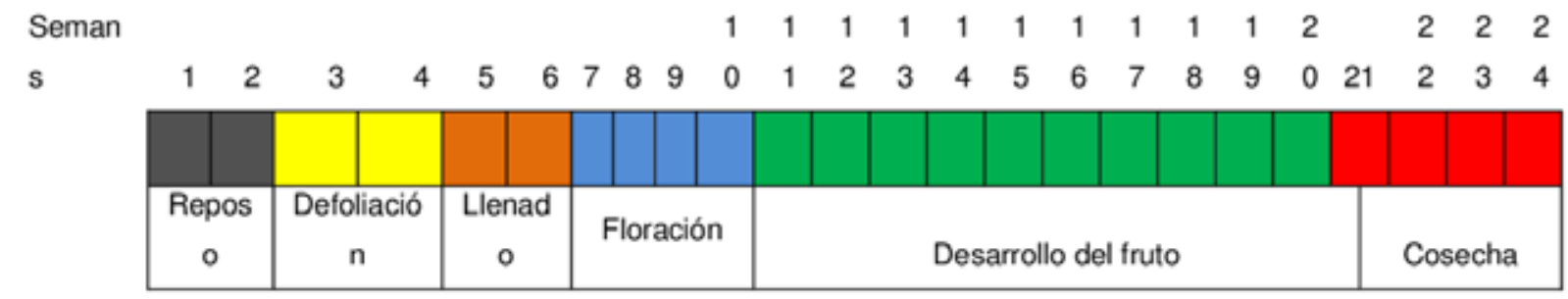

Fuente: Elaboración del autor (2016).

Figura 4. Programa de producción actual, cinco fincas

\begin{tabular}{|c|c|c|c|c|c|c|c|}
\hline \multirow[b]{2}{*}{ Fincas } & \multirow[b]{2}{*}{ NPlantas } & \multicolumn{6}{|c|}{ Primera producción } \\
\hline & & Enero & Febrero & Marzo & Abril & Mayo & Junio \\
\hline Finca 1 & 250 & & & & & & \\
\hline Finca 2 & 200 & & & & & & \\
\hline Finca 3 & 380 & & & & & & \\
\hline Finca 4 & 450 & & & & & & \\
\hline Finca 5 & 100 & & & & & & \\
\hline
\end{tabular}

acuerdo con el estado fenológico del cultivo; se destacan labores como la cosecha, en donde cada obrero recolecta 12 a 15 canastillas/ día. Para elaborar estos cuadros se utiliza el programa Excel, y atendiendo las características del sistema de producción se elaboran; para el ejemplo trabajado, se necesitarían 5 cuadros, uno por finca. La estructura del cuadro se hace teniendo en cuenta los ciclos de producción, la duración de los estados fenológicos si los hay, y las labores culturales, el número de jornales, de horas máquina que se asigna depende de los indicadores que se manejen en la región.

Producción de las cinco fincas antes de efectuar la planificación. Antes de iniciar el proceso de planificación de cosechas se pudo observar que las cinco fincas estudiadas presentaron las épocas de cosecha en meses iguales, dando origen a la concentración de la producción (Figura 3); épocas que coinciden con la oferta de cirvela en el país, los meses de junio y diciembre.

En la Figura 4 se muestra que los productores no hacen planificación en la producción, ya que mantenían una producción constante en solo dos meses del año, diciembre y junio. Para este ejemplo en junio, debido a que no realizan cosechas forzadas, no manejan correctamente las labores culturales como la fertilización, esta no la hacen en el estado fenológico adecuado (floración), ni suministran a la planta las cantidades suficientes que esta necesita, no manejan un sistema de riego, no hacen controles fitosanitarios periódicos, este manejo que hacen los productores en forma tradicional los lleva a sacar la producción siempre en las mismas épocas del año.

Planificación de cosechas de ciruela Horvin en cinco fincas. Para lograr el objetivo de desplazar la época de producción de cirvela en cada una de las cinco fincas, fue necesario trabajar con las siguientes variables:

Al efectuar la poda antes de aplicar los compensadores de frío, se observa un ahorro en los productos aplicados (equivalente a 50 horas aproximadamente), completando las horas frío. También se hacen dos aplicaciones foliares con productos ricos en fósforo y potasio, 
las cuales ayudan a la maduración de yemas; estas se hacen en las dos últimas semanas de la cosecha; se recomienda hacer una poda en verde, que consiste en eliminar chupones para que los cargadores de la parte del centro tengan buena ventilación y aireación, actividad que favorece tener yemas mixtas para una excelente producción.

Se debe hacer una fertilización edáfica a la planta en estado de reposo, que sea rica en fósforo para ayudar a estimular las yemas y suspender lentamente el riego, lo que permite que las hojas vayan madurando. Para poder realizar la planificación de cosechas se inició con la primera cosecha de 2013; se inició con el desplazamiento de las labores y aplicaciones después de cada una de las cosechas; en cada una de las fincas fue necesario desplazar la fase del reposo de las plantas, este se hizo progresivamente en cada cosecha de 8 a 10 días hasta lograr un mes y hasta dos meses, para empezar en el primer semestre de 2014 con una producción constante en las fincas estudiadas.

En la siguiente tabla se muestra la programación de cosechas de las fincas para los años 2014 y 2015, contando con cinco meses seguidos en la producción de cirvela Horvin. La primera finca que empieza con la producción es la finca cinco, la segunda producción se obtiene en la tercera finca, la tercera cosecha en la finca cuatro, la cuarta producción en la primera finca y la quinta cosecha en la finca 2, permitiendo tener una producción de ciruela Horvin en diferentes meses del año. Para poder tener producción constante se usó la técnica de las cosechas forzadas en todas las fincas, con un buen plan de fertilización a la planta, haciendo aplicaciones foliares frecuentes, utilizando sistemas de riego adecuados, manejando correctamente las podas de producción y los compensadores de frío. Se aclara que esta planeación sería la ideal; sin embargo, hay variables naturales como lluvias, sequías, heladas o vientos, que no permiten ocasionalmente que las plantas respondan exactamente como se planea.

Esta planeación es necesaria para hacer un proceso de organización en el manejo de los cultivos, con miras a evitar acumulación de cosecha y pérdida del fruto; además de esto es importante tener claro los canales de comercialización, los cuales están orientados en Corabastos, en la ciudad de Bogotá.
Es importante este tipo de estudios puesto que implican varios aspectos positivos para los productores, ya que se generan estrategias de manejo de cultivo, organización concreta del proceso y disminución de pérdidas del producto.

\section{CONCLUSIONES}

En el municipio de Tuta, Boyacá, específicamente en la vereda Río de Piedras, se encuentran cultivares de cirvela Horvin, que se caracterizan por ser plantaciones de edad promedio 10 años, cultivos de minifundio, con áreas inferiores a una fanegada, densidades de siembra que van desde 100 hasta 450 plantas. El manejo del huerto se hace en forma tradicional; sin embargo, se destaca la experiencia y el trabajo de los pequeños productores en este cultivo, notándose que invierten en los insumos necesarios para garantizar el éxito en las cosechas. La cirvela en esta región produce cada 6 meses, logrando dos cosechas anuales o máximo en 13 meses.

El programa de producción y las prácticas culturales asociadas a la producción de cirvela lograron que se diera el desplazamiento de la cosecha, aspecto que se hizo progresivamente, semana por semana, desde el año 2013, razón por la cual se tienen cosechas en diferente mes en las cinco fincas estudiadas; es importante resaltar la importancia que tuvo el hecho de trabajar las técnicas para el ciclado de frutales caducifolios, a través del manejo de cada uno de los estados fenológicos y la aplicación del producto requerido para tal fin, según las recomendaciones de los ingenieros agrónomos.

Se logró demostrar que la planificación de la producción de cirvela es viable en la región, con un trabajo técnico y administrativo que les permita a los pequeños productores hacer la planificación respectiva, con los insumos requeridos en las fechas programadas y con buenas prácticas de manejo. Así el pequeño productor se dio cuenta de que tener producción constante es una ventaja para él como empresario, dado que se mejoraron los ingresos considerablemente, al pasar de recibir $\$ 15.000$ por una canastilla de cirvela a recibir $\$ 40.000$ por la misma, solo que esta es vendida en meses en que la oferta de producto a nivel nacional es baja. 
Una forma de evaluar el agronegocio de la cirvela es mediante el seguimiento de los cultivos, en donde fue necesario implementar una serie de formatos para documentar cada uno de los procesos y las actividades, aspecto que facilitará a los pequeños productores el manejo administrativo, dado que el tener información real de sus gastos y ventas les permitirá más adelante hacer los correctivos necesarios y una mejor toma de decisiones.

\section{REFERENCIAS}

Agronet (2014). Agronet - Estadísticas agropecuarias. Recuperado de https:// www.agronet.gov.co/estadistica/Paginas/ home.aspx

Agronet (2019). Agronet - Estadísticas Agropecuarias. Recuperado de https:// www.agronet.gov.co/estadistica/Paginas/ home.aspx

Asohofrucol (2012). Frutas y hortalizas. Recuperado a partir de http://www. asohofrucol.com.co/RevistaFyH.php

Asohofrucol (2018). Frutas y hortalizas. Recuperado a partir de http://www. asohofrucol.com.co/RevistaFyH.php

Arauz, L., \& Mora, D. (1983). Preliminary evaluation of the postharvest problems in six tropical fruits in Costa Rica. Agronomía Costarricense, 7 (1), 43-53. San José, Costa Rica, Asociación Hortofrutícola de Colombia (ASOHOFRUCOL).

Coronado, A. (2015). Planificación de cosechas en cinco unidades productivas de cirvela Horvin en el municipio de Tuta, Boyacá.

Departamento Administrativo Nacional de Estadística, DANE (2018). Estadísticas del Sector Agropecuario.

Gobernación de Boyacá (2017). Ordenamiento territorial departamental de Boyacá, Productividad del Sector Agropecuario. Recuperado a partir de http://www dapboyaca.gov.co

Gómez, C. A.; Herrera, A. O., y Flórez, V. J. (2015). Efecto de 1-metilciclopropeno y temperatura de almacenamiento en la poscosecha de mandarina (Citrus reticulata L.) var. Arrayana. Revista de la Facultad de Ciencias Agrarias, vol. 47, núm. 2. Recuperado 18 de marzo de 2017 de: http://www.redalyc.org/articulo. oa? id $=382842590003$
Fred, R. D. (2000). Concepts of strategic management. Columbus, Ohio: Merril Publishing Company.

Guerra, G. (1992). Manual de administración de empresas agropecuarias. Agroamérica.

Lázaro, Hilario J. (2011). Momento oportuno de cosecha de la cirvela D'Agen. Instituto Nacional de Tecnología Agropecuaria. Recuperado de https://inta.gob.ar/ documentos/momento-oportuno-decosecha-de-la-cirvela-dagen

Miranda, D.; Fischer, G., y Carranza, C. (2013). Los frutales caducifolios. Situación actual, sistemas de cultivo y plan de desarrollo en Colombia, 234.Recuperado de file:///C:/Users/USER/Downloads/ LosfrutalescaducifoliosenColombia.pdf

Organización de las Naciones Unidas para la Alimentación y la Agricultura (FAO). (s. f.) Evaluación de las pérdidas. Recuperado de http://www.fao.org/docrep/x5037s/ x5037S03.htm

Orjuela, M. (2016a). Incidencia de las características edáficas y climáticas en el desarrollo del fruto de cirvela variedad Horvin, en el municipio de Nuevo Colón, Boyacá. Maestría, Universidad Nacional de Colombia. Recuperado de http://bdigital.unal.edu.co/55666/7/ MayerlinOrjuelaAngulo.2016.pdf

Orjuela, M. (2016b). Incidencia de las características edáficas y climáticas en el desarrollo del fruto de cirvela variedad Horvin, en el municipio de Nuevo Colón Boyacá. Universidad Nacional de Colombia, Facultad de Ingeniería, Departamento de Ingeniería Civil y Agrícola, Bogotá.

Ortiz M., R., R.; Grasso, R., y P. C. (2016). Evaluación de alternativas de manejo de poscosecha en hortalizas de hoja sobre las pérdidas a nivel minorista. Revista FAVE - Ciencias Agrarias 13 (1 - 2). DOI:10.14409/ fa.v13i1/2.4961

Parra, A.; Hernández, J. y Camacho, J. (2007). Estudio de algunas propiedades físicas y fisiológicas precosecha de la cirvela variedad Horvin. Revista Brasileira de Fruticultura, 29(3),431-437. https://dx.doi. org/10.1590/S0100-29452007000300006. 
Puentes, G. A. (2006). Sistema de producción de frutales caducifolios en el departamento de Boyacá. Equidad y Desarrollo, (5), 39-46.

Puentes, G. A.; Prieto, D. F., y Caro, L. S. (2019). Formulación y evaluación de proyectos agropecuarios. Estructura del proyecto agropecuario, con enfoque de marco lógico. (ECO ediciones, ed.) (2ºdición).

Ramírez, M. R.; Jacobo, J. L.; Ávila, M. R., y Parra, R. Á. (2006). Pérdidas de cosecha, eficiencia de producción y rentabilidad de huertos de manzano con diversos grados de tecnificación en Chihuahua, México. Revista Fitotecnia Mexicana. vol. 29, núm. 3. Recuperado 10 de marzo de 2017: http:// www.redalyc.org/articulo.oa? id=61029305
Rozo, R.; Álvarez, J. G. Á., y Reyes, A. (2015). Comportamiento poscosecha de frutos de cirvela (Prunus salicina Lindl.) en cuatro estados de madurez tratados con etileno. Revista Colombiana de Ciencias Hortícolas, Vol. 9, No. 1, 46-59. https://doi.org/Doi: http:// dx.doi.org/10.17584/rcch.2015v9i1.3745

Sánchez, Pe (2015). Influencia de la temperatura en etapas postcosecha sobre la calidad de diferentes variedades de cirvela. Universidad Politécnica de Valencia. Recuperado de https://riunet.upv.es/bitstream/handle/

(C) $(1) \Theta \Theta$ 